UDK 528.946

\title{
PRODUCTIVITY INDEX MODELING OF ASMARI RESERVOIR ROCK USING GEOSTATISTICAL AND NEURAL NETWORKS METHODS (SW IRAN)
}

\author{
Hakimeh AMANIPOOR \\ Khorramshahr University of Marine Science and Technology, Khorramshahr, Iran \\ E-mail:h_amanipoor@yahoo.com
}

Received 3 May 2017; accepted 16 August 2017

\begin{abstract}
In this study, productivity index in a carbonate reservoir was predicted using Artificial Neural Networks and geostatistical method. At first, about 518 data of productivity index based on locations of the wellbores were used for modeling and then 40 data were used for investigating the accuracy of the models. Then, the result of ANN was compared with the output of geostatistical modeling. The study shows that productivity index could be estimated with these methods with accepted accuracy. In addition, both modeling have almost the same result. However, accuracy of the geostatistical model by taking into account the spatial structure, is higher than that of neural network.
\end{abstract}

Keywords: carbonate reservoir, productivity index, artificial neural network, geostatistics.

\section{Introduction}

In reservoir engineering, some issues do not obey mathematical formula and models. This is due to the fact that reservoirs are underground and knowing them completely is impossible (Abass et al. 2006). Whatever, a reservoir is more unknown; the prediction of its behavior becomes more difficult. So, some modelings apart from mathematics are required to simulate some special properties of reservoirs. Statistics is helpful and easy to use, but due to high dependency of the parameter on location, geostatistics is the best method for modeling this type of data. Artificial Neural Network (ANN) is another method for modeling this type of parameter. An ANN is a system of simple processing units known as neurons or nodes. These elements are linked with each other through simple connections known as synaptic connections. Neurons are organized in layers. In a back propagation ANN (BP-ANN), the network output is subtracted from the actual or desired output of the problem. The resulting error is then propagated through the network and weights are adjusted accordingly. This process, named training, continues until the network output reaches an acceptable value (Mohaghegh et al. 1994; Bean, Jutten 2000).

Productivity index (PI) is an important parameter for petroleum engineers. It plays an important role in different stages of a well, from drilling to production. It is a typical measure of well deliverability, reservoir permeability, skin, drawdown pressure and so on. If production index of a location to be drilled is known before beginning the drilling operation, mud design and preparation could be done in an easier way. Productivity index for production is also very important.

\section{Problem statement}

In a carbonate oil reservoir, the productivity index from buildup tests is available. To find new location for oil producing well with high productivity index and also to investigate the condition of predefined drilling location for mud design purposes, a study on available PI data was conducted. The purpose is to predict PI for locations in which no data is available. Locations of data for modeling and testing the accuracy of the prepared models are shown in Figure 1.

\section{Geological setting}

The studied oilfield is located in the southwestern part of Iran and the eastern side of the great basin of Dezfoul embayment. The dimensions of the field are approximately $70 \mathrm{~km}$ length and $15 \mathrm{~km}$ width, the distance between the reservoir crest and the deepest 


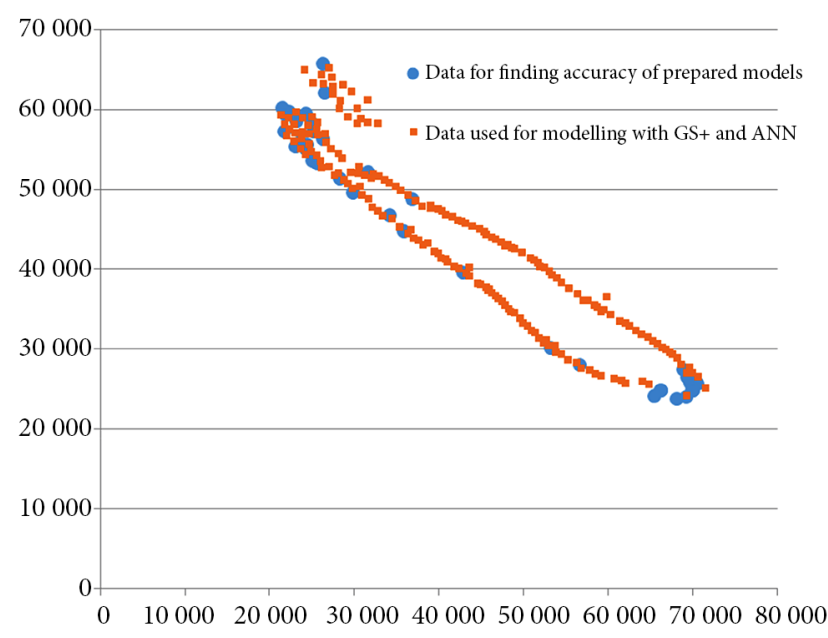

Fig. 1. Location of wellbores in the field

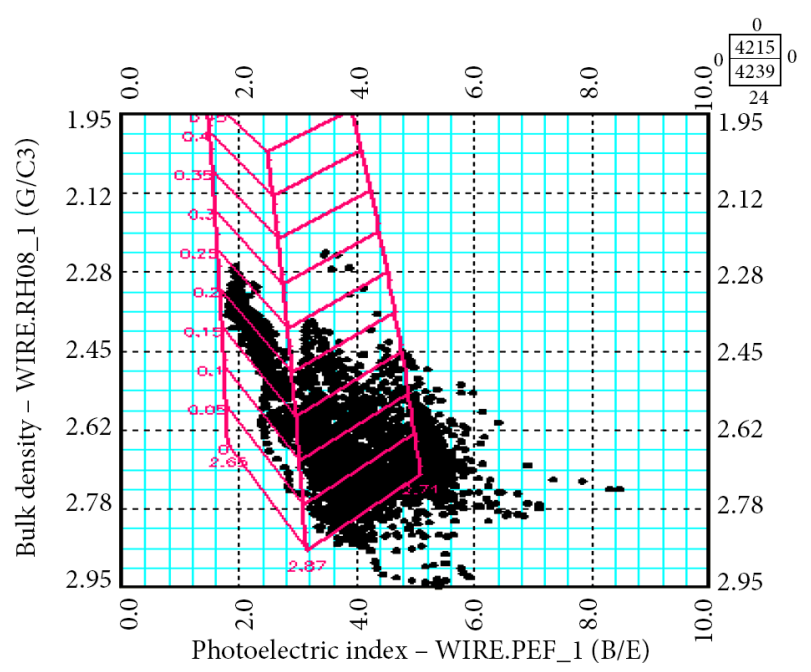

Fig. 2. PEF-RHOB cross plot (represents carbonate and sandstone lithology)

water and oil interface reservoir in Asmari Formation is about $2000 \mathrm{~m}$.

Lithology is one of the important parameters in recognition of hydrocarbon reservoir's behavior. It can be identified using petrophysical data (Ellis, Singer 2007). The main lithology of this reservoir are carbonate and to a lesser extent, sandstones (Fig. 2).

\section{Spatial modeling}

There are several methods for modeling and estimating of data. Most of these methods are based on statistical and geostatistical principles. Classical methods are those that use classical statistics for estimation, while in geostatistical methods, the estimation is made based on spatial structure in the environment (Abdideh, Abyat 2012; Amanipoor 2013). Variogram analysis is an important part of geostatistical modeling. Actually, variogram, which calculated using the following equation, shows the mean square difference between two values as a function of their increment (Isaak, Srivastava 1989):

$$
\gamma(h)=\frac{\sum_{i=1}^{n_{h}}\left(x_{i}-x_{i+h}\right)^{2}}{2 n_{h}},
$$

where $\gamma(h)$ : semivariogram $(2 \gamma(h)$ : variogram $), x_{i}$ : value in point $i, x_{i+h}$ : value in a point with distance $h$ from the point $i, n_{h}$ : the number of pairs of points with the distance $h$ from each of them participating. The $\gamma(h)$ diagram can be drawn using calculating variogram for different $h$ values, which is called lag.

In most cases, the variogram starts from a nonzero value and increases up to a value that called effective range (a), eventually reaching the constant value. The effective range is the range in which the data spatial structure is relevant, and outside this range, the data effect is independent of each other (Corstange et al. 2008).

Theoretically, the variogram value at the starting point $(h=0)$ should has its minimum value (i.e. zero), but in practices, this function, which is the result of experience, does not usually follow this condition. The variogram value for $h=0$ is called Nugget effect. As $h$ increases, variogram starts from low values and tends towards a constant limit that is called sill. Changes of sill are only by accident. Therefore, some variograms reach a relatively constant value and after that, with any increase in distance, the amount of variogram does not change significantly. In geostatistics, variograms reaching $a$ constant sill are more important and they are appropriate for estimation. To calculate and plot experimental variograms and before using experimental variograms in estimation, the most appropriate theoretical model should be fitted (Ortiz, Deutsch 2002). There are several theoretical models for fitting to experimental variograms.

In general, geostatistical estimation is a process in which the value of a quantity in points with known coordinates can be determined using the value of the same quantity at different points with known coordinates. Kriging is an estimation method based on a weighted moving average (equation 2), and it can be regarded as the Best Linear Unbiased Estimator (BLUE) (Abdideh, Bargahi 2012):

$$
Z_{v}^{*}=\sum_{i=1}^{n} \lambda_{i} z_{v i}
$$

where $Z_{v}^{*}$ - estimation assay, $z_{v i}$ - the assay of ith sample, $\lambda_{i}$ - weight or importance of the quantity dependent on the ith sample. This type of kriging, which is called linear kriging, is a linear combination of $n$ 
data. This estimator is used when the variable $Z$ has normal distribution. If the variable is not normally distributed, non-linear Kriging is used, or first a transformation that transforms the distribution of the desired variable to normal is found, and then linear Kriging is performed on transformed data (Hassani Pak 2013).

The most important feature of Kriging is that for each error estimation, the relevant associated error can be calculated. Therefore, for each estimated value, the relevant confidence range can be calculated (Deutsch 2002). Some of the Kriging characteristics are (Abdideh 2014):

- Kriging accompanied by any estimation, reduces its error, and not only can the average error be calculated, but also, the error distribution (estimation variance) can be determined in the whole considered area under study.

- If the quantitative value is estimated in sampling points, the estimated value is equal to the measured value, and the estimation variance becomes zero.

- Kriging is a function of variogram characteristics of estimated blocks and the geometry of blocks used for estimation, but it is not a function of true value of data.

- Kriging causes softening of changes (reduced vibrations), that is, the assay distribution of the estimated blocks relative to true assay of blocks has slight changes.

\section{Results and discussion}

First, 518 data were obtained from PI test of the reservoir. Some wells had more than one result and geostatistical method was used to calculate their mean. In addition, 40 data were deleted randomly for investigation of the accuracy of the prepared models.

Histograms and accumulative frequency provide beneficial information on the variation and statistical parameters of the studied parameter. Normality or abnormality of the data was investigated and after normalization of data, variogram was drawn so that calculation and selection of the best variogram and extraction of required data from variogram could be done for appropriate estimation. Variogram shows structural analysis and spatial modeling of porosity parameter in sampling environment.

As the logarithm has more meaningful relation between different parameters in petroleum engineering, in this study, logarithm of PI was used as input for modeling in addition to northing and easting. The

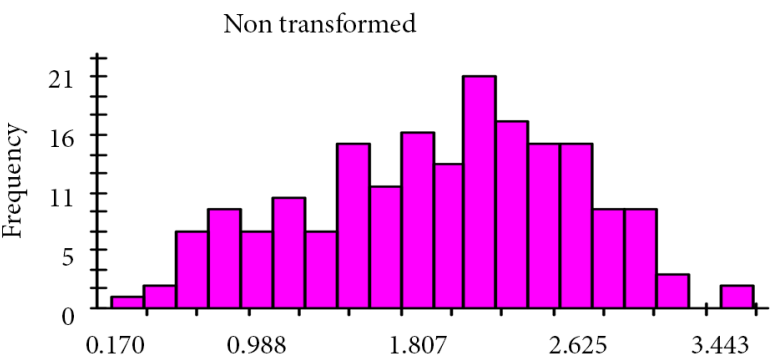

Fig. 3. Distribution graph of PI parameters (represents the distribution is almost normal)

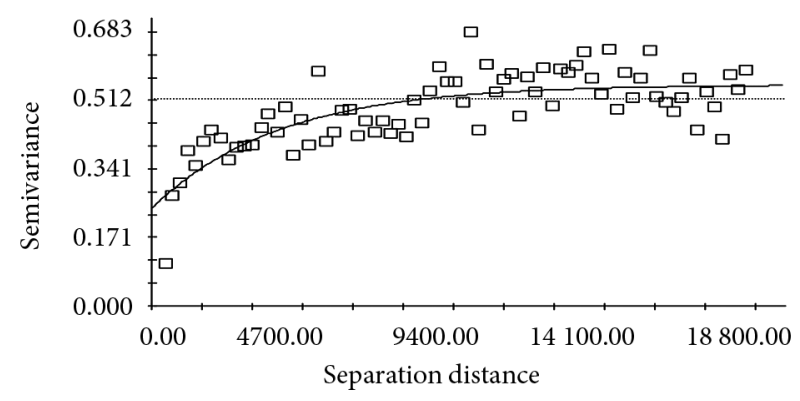

Exponential model $\left(\mathrm{C}_{0}=0.2427 ; \mathrm{C}_{0}+\mathrm{C}=0.5514 ; \mathrm{A}_{0}=3930.00, \mathrm{r}_{2}=\right.$ 0.606, RSS $=0.254$ )

Fig. 4. Variogram model for PI data

almost normal distribution of input data shown in Figure 3 is related to this subject.

To match isotropic variogram, active lag distance and lag class distance interval were chosen to be 18800 and 252, respectively. Isotropic variogram model is shown in Figure 4.

In continuation, calculation of anisotropic variograms for angles from 0 to $175^{\circ}$ with increase of $15^{\circ}$ and tolerance of 15-30-45-60-75-90, was done. To find the best model, some parameters were analyzed. These parameters are residual sum of squares (RSS), regression coefficient $\left(\mathrm{R}^{2}\right)$ and the fraction of Sill/ (Sill+Nugget). The smaller the nugget is better model and makes the fraction to increase to 1 , although, this is not enough to select the best model. RSS and $\mathrm{R}^{2} \mathrm{~Pa}$ rameters are more important.

The RSS parameter is the sum of squares of the difference between the actual and estimated values. Therefore, the lower value of this parameter indicates a more accurate estimate and a better model fit. In geostatistical software, the RSS is used to choose parameters for each of the variogram using determining the combination of parameter values that minimizes RSS for any given model. The $\mathrm{R}^{2}$ parameter also shows how well the model fits the variogram data. Although for best model selection, this parameter is not robust or sensitive such as the RSS value. Finally the best model calculated by geostatistical software selected based on min RSS (Gringurten, Deutsch 1999). 
After specification of spatial structure by variogram analysis, PI parameter in the whole reservoir layer was estimated. One of the best methods for preparation of distribution maps is the Kriging method.

Kriging is the best linear unbiased estimator with the least estimation variance, which together with any estimation, yields its error value; so, it is possible not only to calculate average value of error but also to study the error distribution in the complete studied range. Kriging is an estimation method that is based on weighted moving average. Kriging is used as a mapping algorithm and is a low stream filter inclined towards smoothing small and large amounts and values of initial raw data.

Figure 5 is an estimation map of PI parameter in the studied reservoir using Kriging method. Calculation was done for all locations using Kriging Estimator. The cross validate graph and Kriging map are shown in Figure 6.

Finally, to investigate the accuracy of the model, by using northing and easting of deleted data, the estimated value of log (PI) was read from Kriging map.

\section{Simulation with ANN}

In continuation, the same data was used for simulating by ANN method. There are many parameters for

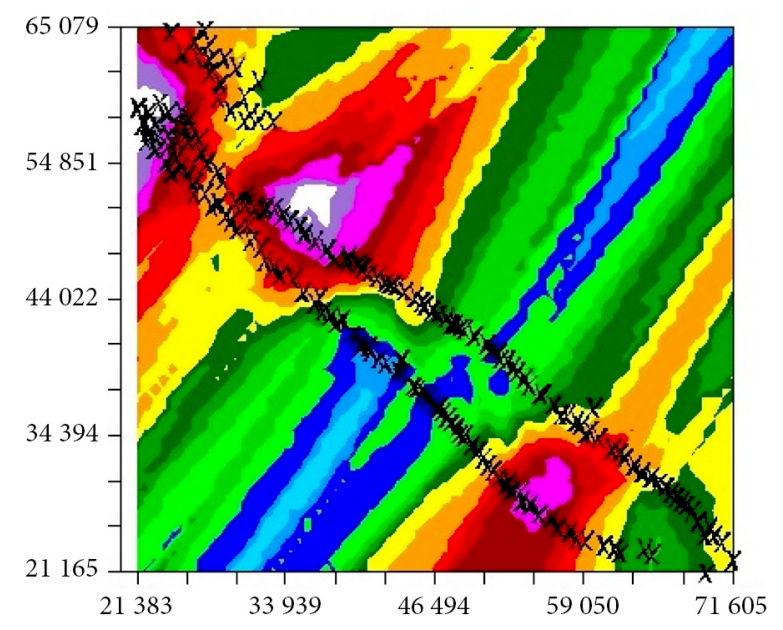

Fig. 5. Estimation of PI distribution by Kriging method

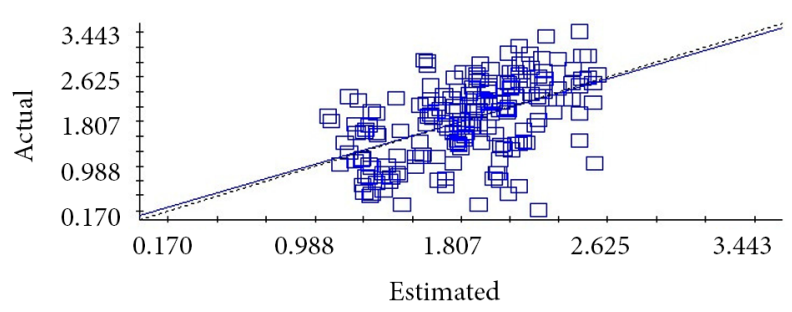

Fig. 6. Validation of PI distribution estimation by Kriging method investigating power of modeling by ANN and also for matching the data, but in this study, only a few options were selected to compare the geostatistical modeling results. Different options like training, adaption-learning and performance functions and network type are available.

Similar to work done by geostatistical modeling, 40 data were deleted to test the accuracy of ANN model. Then, by choosing the desired options, neurons were created and educated. A sample cross validation graph was created using neural network tool as shown in Figure 7.

Then, to compare the result of both methods, by using deleted PI data, their value were estimated by ANN.

The real value of $\log (\mathrm{PI})$ for the deleted wellbores, and the estimated one for both methods are shown in Table 1. The difference between the real and estimated data was also calculated. It is shown that the percent of difference between real and estimated data for geostatistical method and ANN, respectively varies from 0.079 to $270.262 \%$ and 0.5 to $318 \%$ with a mean of 35.9 and $36.5 \%$, respectively.

Table 1. Compare the two methods of geostatistical and ANN for estimation PI parameter

\begin{tabular}{|c|c|c|c|c|c|c|c|}
\hline \multirow[t]{2}{*}{ 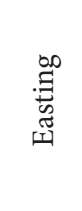 } & \multirow[t]{2}{*}{ 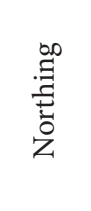 } & \multirow[t]{2}{*}{ 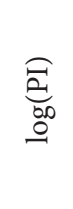 } & \multirow[t]{2}{*}{$\bar{a}$} & \multicolumn{2}{|c|}{$\begin{array}{c}\text { Estimation } \\
\text { of } \log (\mathrm{PI})\end{array}$} & \multicolumn{2}{|c|}{$\begin{array}{c}\text { \% Difference } \\
\text { between Real \& } \\
\text { Estimated } \\
\log (\mathrm{PI})\end{array}$} \\
\hline & & & & GS & ANN & GS & ANN \\
\hline 1 & 2 & 3 & 4 & 5 & 6 & 7 & 8 \\
\hline 69627 & 25762 & 1.736 & 55 & 1.73 & 1.76 & 0.368 & 1.2 \\
\hline 21482 & 60159 & 2.523 & 333 & 2.54 & 2.76 & 0.679 & 9.5 \\
\hline 66237 & 24899 & 1.745 & 56 & 1.52 & 1.52 & 12.882 & 12.9 \\
\hline 25026 & 53623 & 3.301 & 2000 & 2.15 & 2.29 & 34.869 & 30.5 \\
\hline 66173 & 24925 & 1.726 & 53 & 1.52 & 1.51 & 11.927 & 12.3 \\
\hline 25617 & 53242 & 3.000 & 1000 & 2.12 & 2.13 & 29.333 & 29.1 \\
\hline 26507 & 62070 & 2.125 & 133 & 2.05 & 2.02 & 3.527 & 4.8 \\
\hline 24401 & 55630 & 3.301 & 2000 & 2.21 & 2.41 & 33.051 & 27.0 \\
\hline 69219 & 24062 & 2.314 & 206 & 1.66 & 1.79 & 28.259 & 22.7 \\
\hline 23150 & 58578 & 2.222 & 167 & 2.53 & 2.56 & 13.869 & 15.0 \\
\hline 25110 & 58187 & 2.301 & 200 & 2.26 & 2.20 & 1.783 & 4.5 \\
\hline 70597 & 25735 & 3.067 & 1167 & 1.75 & 1.82 & 42.940 & 40.6 \\
\hline 35810 & 44774 & 2.301 & 200 & 1.89 & 1.87 & 17.863 & 18.8 \\
\hline 29899 & 49575 & 0.863 & 7 & 1.91 & 0.94 & 121.249 & 8.9 \\
\hline 24275 & 59453 & 2.699 & 500 & 2.49 & 2.31 & 7.743 & 14.5 \\
\hline 65446 & 24147 & 1.602 & 40 & 1.56 & 1.50 & 2.625 & 6.4 \\
\hline
\end{tabular}


Continued Table 1

End of Table 1

\begin{tabular}{|c|c|c|c|c|c|c|c|}
\hline 1 & 2 & 3 & 4 & 5 & 6 & 7 & 8 \\
\hline 22288 & 59731 & 2.602 & 400 & 2.6 & 2.65 & 0.079 & 1.7 \\
\hline 68159 & 23834 & 1.174 & 15 & 1.61 & 1.73 & 37.148 & 47.1 \\
\hline 69921 & 24875 & 2.273 & 188 & 1.72 & 1.80 & 24.329 & 20.6 \\
\hline 69981 & 24927 & 2.648 & 444 & 1.73 & 1.81 & 34.663 & 31.8 \\
\hline 69954 & 24829 & 1.083 & 12 & 1.72 & 1.81 & 58.867 & 67.0 \\
\hline 24292 & 54979 & 2.071 & 118 & 2.23 & 2.46 & 7.699 & 18.9 \\
\hline 21727 & 57280 & 0.697 & 5 & 2.58 & 2.91 & 270.262 & 318.0 \\
\hline 70014 & 24870 & 1.252 & 18 & 1.73 & 1.81 & 38.200 & 44.6 \\
\hline 28258 & 51319 & 3.000 & 1000 & 1.89 & 1.31 & 37.000 & 56.2 \\
\hline 26252 & 56346 & 1.041 & 11 & 2.09 & 1.99 & 100.776 & 90.9 \\
\hline 25150 & 57278 & 3.000 & 1000 & 2.17 & 2.20 & 27.667 & 26.7 \\
\hline 22710 & 59220 & 1.685 & 48 & 2.59 & 2.60 & 53.734 & 54.5 \\
\hline 23092 & 55358 & 3.000 & 1000 & 2.36 & 2.74 & 21.333 & 8.8 \\
\hline 26239 & 65762 & 2.398 & 250 & 1.99 & 1.77 & 17.012 & 26.3 \\
\hline 36837 & 48784 & 2.456 & 286 & 2.38 & 2.47 & 3.092 & 0.5 \\
\hline 23551 & 55782 & 2.222 & 167 & 2.32 & 2.61 & 4.418 & 17.6 \\
\hline 69386 & 26457 & 2.243 & 175 & 1.72 & 1.72 & 23.318 & 23.3 \\
\hline 34223 & 46817 & 2.699 & 500 & 2.09 & 2.32 & 22.563 & 14.1 \\
\hline 42863 & 39577 & 1.114 & 13 & 1.13 & 0.96 & 1.481 & 13.8 \\
\hline
\end{tabular}

\begin{tabular}{|c|c|c|c|c|c|c|c|}
\hline 1 & 2 & 3 & 4 & 5 & 6 & 7 & 8 \\
\hline 21823 & 59386 & 2.699 & 500 & 2.6 & 2.76 & 3.667 & 2.3 \\
\hline 53203 & 30124 & 2.097 & 125 & 2.27 & 2.58 & 8.255 & 23.0 \\
\hline 68853 & 27422 & 2.523 & 333 & 1.67 & 1.66 & 33.805 & 34.1 \\
\hline 31637 & 52177 & 2.699 & 500 & 2.2 & 2.29 & 18.487 & 15.0 \\
\hline 56652 & 27999 & 0.690 & 5 & 2.24 & 2.39 & 224.464 & 245.6 \\
\hline & & & $\begin{array}{c}\text { Min of } \\
\% \text { of } \\
\text { Difference }\end{array}$ & 0.079 & 0.5 \\
\hline & & $\begin{array}{c}\text { Max of } \\
\text { \% of } \\
\text { Difference }\end{array}$ & 270.262 & 318 \\
\hline & & $\begin{array}{c}\text { Mean } \\
\text { of \% of } \\
\text { Difference }\end{array}$ & 35.9 & 36.5 \\
\hline
\end{tabular}

\section{Conclusions}

Common methods of location-based parameters are geostatistical methods and neural networks. In this paper, these two methods were compared to estimate reservoir parameters.

By using geostatistical method, a good model for predicting PI of a carbonate reservoir was obtained.
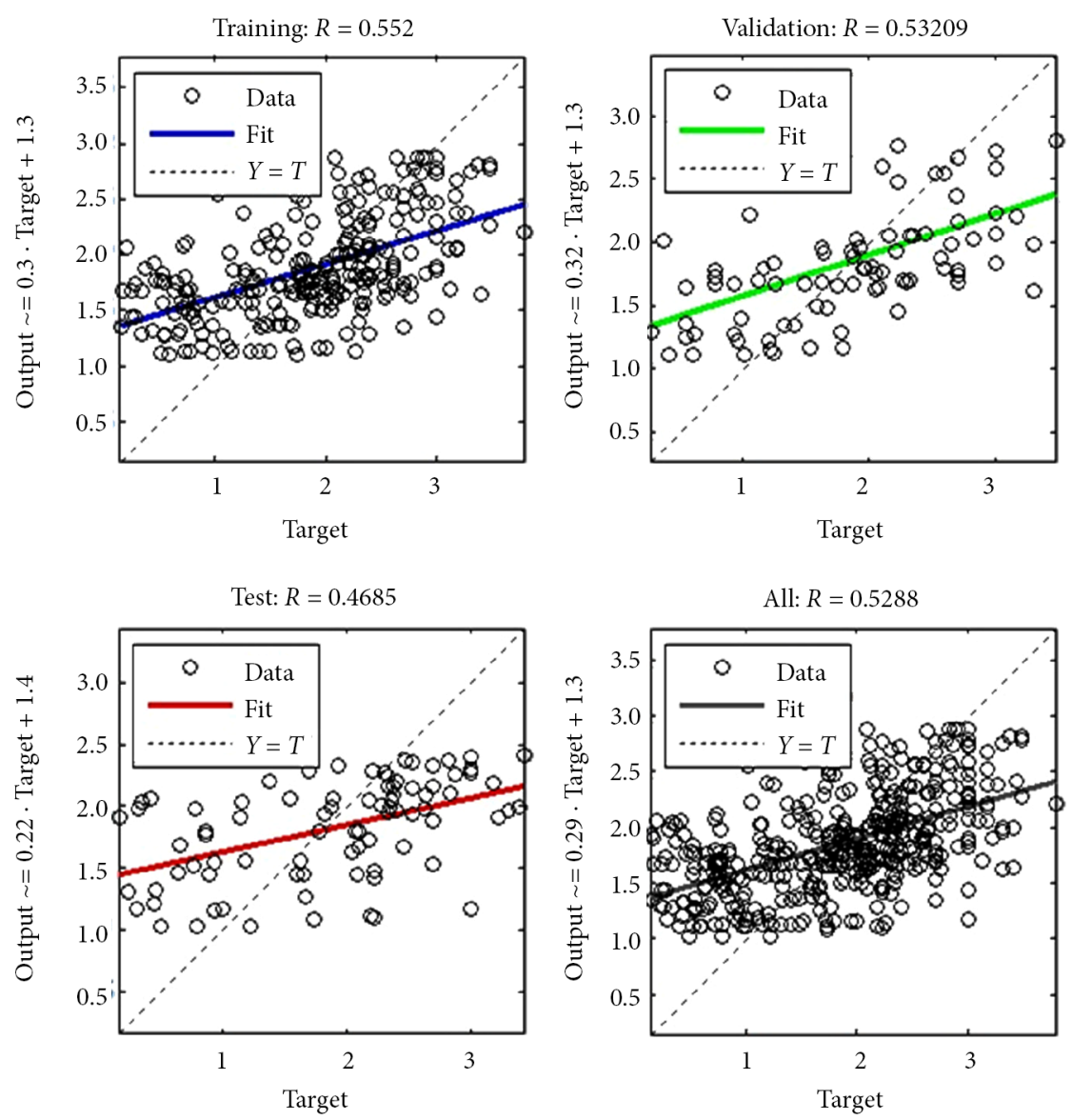

Fig. 7. Cross validate graph by ANN 
Although, this model is not very accurate, in the parts where the number of input data is high enough, the estimation becomes more accurate. For example, from 40 data tested for accuracy, in 18 , the error was below $20 \%$ and in 25 , the error was less than $30 \%$.

In conclusion, the results of ANN and geostatistical models match each other and their accuracy is almost similar. It means that both have the same order of error.

\section{Acknowledgement}

We would like to thank Khorramshahr University of Marine Science and Technology for supporting this work under research grant contract No 135.

\section{References}

Abass, H.; Shebatalhamd, A.; Khan, M.; Al-Shobaili, Y.; Ansari, A.; Ali, S.; Mehta, S. 2006. Wellbore instability of shale formation; Zuluf field, in SPE Technical Symposium of Saudi Arabia Section, 21-23 May 2006, Dhahran, Saudi Arabia. https://doi.org/10.2118/106345-MS

Abdideh, M. 2014. Prediction of mud loss in reservoir rock by geostatistical method, Geomatics, Natural Hazards and Risk 5: 41-55. https://doi.org/10.1080/19475705.2013.773944

Abdideh, M.; Abyat, M. 2012, A geostatistical approach for predicting the top producing formation in oil fields, Geodesy and Cartography 38(3): 111-117.

https://doi.org/10.3846/20296991.2012.728896

Abdideh, M.; Bargahi, D. 2012. Designing a 3D model for the prediction of the top of formation in oil fields using geostatistical methods, Geocarto International 27: 569-579. https://doi.org/10.1080/10106049.2012.662529
Amanipoor, H. 2013. Providing a subsurface reservoir quality maps in oil fields by geostatistical methods, Geodesy and Cartography 39(4): 145-148.

https://doi.org/10.3846/20296991.2013.859779

Bean, M.; Jutten, C. 2000. Neural networks in geophysical applications, Geophysics 65: 1032-1047. https://doi.org/10.1190/1.1444797

Corstange, R.; Grunwald, S.; Lark, R. M. 2008. Inferences from fluctuations in the Local Variogram about the assumption of stationary in the variance, Geoderma 143: 123-132. https://doi.org/10.1016/j.geoderma.2007.10.021

Deutsch, C. V. 2002. Geostatistical reservoir modeling. Oxford: Oxford University Press. 376 p.

Ellis, D. V.; Singer, J. M. 2007. Well logging for earth scientists. Dordrecht: Springer-Verlag. https://doi.org/10.1007/978-1-4020-4602-5

Gringurten, E.; Deutsch, C. 1999. Methodology for variogram interpretation and modeling for improved reservoir characterization, in SPE Annual Technical Conference and Exhibition, 3-6 October 1999, Houston, Texas, USA. https://doi.org/10.2118/56654-MS

Hassani Pak, A. A. 2013. Geostatistical. 5th ed. Tehran: University of Tehran press. 328 p. (in Persian).

Isaak, E. H.; Srivastava, R. M. 1989. An introduction to applied geostatistical. Vol. 561. Oxford: Oxford University Press.

Mohaghegh, S.; Arefi, R.; Ameri, S.; Rose, D. 1994. Design and development of an artificial neural network for estimation of permeability. SPE 28237, Society of Petroleum Engineers.

Ortiz, J.; Deutsch, C. V. 2002. Calculation of uncertainty in the variogram, Mathematical Geology 34(2): 169-183. https://doi.org/10.1023/A:1014412218427

Hakimeh AMANIPOOR. Assistant Professor of Engineering Geology. Department of Environment, Natural Resources Faculty, Khorramshahr University of Marine Science and Technology, Khorramshahr, Iran. Research interests: engineering geology, environmental geology, Geostatistical modelling, petrophysics. 